\title{
ANOTHER GENERALIZATION OF BROUWER'S FIXED POINT THEOREM
}

\section{DAVID HENDERSON AND G. R. LIVESAY}

The theorem proved here is naturally suggested by the following observation. If $D^{n}$ is a closed $n$-cell in euclidean $n$-space, $E^{n}$, with boundary $S^{n-1}$ embedded nicely enough so that there is a retraction of the closure of $E^{n}-D^{n}$ onto $S^{n-1}$, then any map $f:\left(D^{n}, S^{n-1}\right)$ $\rightarrow\left(E^{n}, D^{n}\right)$ has a fixed point. This is an immediate consequence of the Brouwer fixed point theorem. R. H. Bing, [1], has shown that there is always a retraction, $r$, of the closure of the unbounded component of $E^{n}-S^{n-1}$ onto $S^{n-1}$, no matter how badly $S^{n-1}$ is imbedded in $E^{n}$. Hence the Brouwer fixed point theorem may be generalized by applying the Lefschetz fixed point theorem to the map $\bar{r} \cdot f$, where $f:\left(Y, S^{n-1}\right) \rightarrow\left(E^{n}, Y\right), Y$ is the closure of the bounded component of $E^{n}-S^{n-1}, \bar{r}:\left(E^{n}, Y\right) \rightarrow(Y, Y)$ is defined by $\bar{r} \mid Y=1$, and $\bar{r} \mid \mathrm{Cl}\left(E^{n}-Y\right)$ $=r$ is Bing's retraction onto $S^{n-1}$. Then observe that a fixed point of $\bar{r} f$ is a fixed point of $f$. In fact, Bing has a more general retraction theorem which suggests the following extension of the Brouwer fixed point theorem.

TheOREM. Let $X$ be an $(n-2)$-connected, compact $A N R$ which is contained in a compact, metrizable n-manifold, $M$, which is a rational homology $n$-sphere. If $E$ is a component of $M-X$ and $p \in E$, then each map $f:(M-E, X) \rightarrow(M-p, M-E)$ has a fixed point.

Proof. By Theorem 3 of [1], there is a retraction, $r$, of $(E \cup X)-p$ onto $X$. Define a new map $r^{*}: M-p \rightarrow M-E$ by setting $r^{*}(x)=r(x)$ for $x \in(E \cup X)-p$, and $r^{*}(x)=x$ for $x \in M-E$. Suppose that $r^{*} f(x)=x$ for some point in $M-E$. If $f(x) \in E$, then $x=r^{*} f(x) \in X$, which is impossible since $f(X) \subset M-E$. Therefore $f(x) \in M-E$, and $r^{*} f(x)=f(x)=x$. Thus the theorem will be proved if we can show that each map of $M-E$ into itself has a fixed point.

Since $M$ is a metrizable manifold, it is an ANR ([4, p. 98]); thus $M-E$, being a retract of $M-p$, is an ANR $([4$, p. 97]). The Lefschetz fixed point theorem applies to ANR's (see [2, p. 322]) and therefore it suffices to show that $M-E$ is acyclic $([2$, p. 326]). We shall use Cech and singular homology theories with rational coefficients, $Q$. (These theories coincide for compact ANR's ([4, p. 145]).) Let $N$ be a homologically trivial ANR such that $M-E \subset N \subset M$. (For instance,

\footnotetext{
Received by the editors November 28, 1966.
} 
let $M-N$ be the interior of a small $n$-cell containing p.) Since $X$ is $(n-2)$-connected, $H_{i}(X)=0$ for $0<i \leqq n-2$ and $H_{0}(X) \cong Q \quad([\mathbf{5}$, p. 349]). In Cech homology theory on the category of compact pairs every triad is a proper triad ([3, p. 266]). Therefore, we may apply the Mayer-Vietoris sequence to the triad $(N,(E \cup X) \cap N, M-E)$ and conclude that $H_{i}(M-E)=0$, for $0<i \leqq n-2$, and $H_{0}(M-E) \cong Q$. Since $M-E$ is a proper subset of a connected $n$-manifold, it follows that $H_{i}(M-E)=0$ for $i \geqq n$. (We assume here that $n>0$. If $n=0$, the theorem is trivial.) By Alexander duality, (see [6, p. 263]) since $E$ is arc-wise connected, $H_{n-1}(M-E)=0$. Therefore $M-E$ is acyclic and the theorem follows.

\section{REFERENCES}

1. R. H. Bing, Retractions onto spheres, Amer. Math. Monthly 71 (1964), 481-484.

2. S. Lefschetz, Algebraic topology, Amer. Math. Soc. Colloq. Publ., Vol. 27, Amer. Math. Soc., Providence, R. I., 1942.

3. S. Eilenberg, and N. Steenrod, Foundations of algebraic topology, Princeton, Univ. Press, Princeton, N. J., 1952.

4. S.-T. Hu, Theory of retracts, Wayne State Univ. Press, Detroit, Mich., 1965.

5. P. J. Hilton and S. Wylie, Homology theory, Cambridge Univ. Press, Cambridge, 1962.

6. R. L. Wilder, Topology of manifolds, Amer. Math. Soc. Colloq. Publ., Vol. 32, Amer. Math. Soc., Providence, R. I., 1949.

Cornell University

\section{A $T_{1}$-COMPLEMENT FOR THE REALS}

\section{A. K. STEINER AND E. F. STEINER ${ }^{1}$}

The family of all topologies definable on an arbitrary set $X$ forms a complete lattice $\Sigma$ under the partial ordering: $\tau_{1} \leqq \tau_{2}$ if and only if $\tau_{1} \subseteq \tau_{2}$. The lattice operations $\wedge$ and $\vee$ are defined as: $\tau_{1} \wedge \tau_{2}=\tau_{1} \cap \tau_{2}$ and $\tau_{1} \bigvee \tau_{2}$ is the topology generated by the base $\beta=\left\{B: B=U_{1} \cap U_{2}\right.$, $U_{1} \in \tau_{1}$ and $\left.U_{2} \in \tau_{2}\right\}$. The greatest element, 1 , is the discrete topology and the least element, 0 , is the trivial topology. The lattice $\Sigma$ has been recently studied [2], [3], [4] and has been shown to be complemented [4].

Received by the editors October 31, 1966.

${ }^{1}$ Research partially supported by the National Science Foundation, Grant GP 6529 . 